\title{
Research Article \\ Optimal Airport Surface Traffic Planning Using Mixed-Integer Linear Programming
}

\author{
P. C. Roling and H. G. Visser \\ Faculty of Aerospace Engineering, Delft University of Technology, P.O. Box 5058, 2600 GB Delft, The Netherlands
}

Correspondence should be addressed to P. C. Roling, p.c.roling@tudelft.nl

Received 16 May 2007; Revised 24 February 2008; Accepted 2 April 2008

Recommended by K. Sudhakar

\begin{abstract}
We describe an ongoing research effort pertaining to the development of a surface traffic automation system that will help controllers to better coordinate surface traffic movements related to arrival and departure traffic. More specifically, we describe the concept for a taxi-planning support tool that aims to optimize the routing and scheduling of airport surface traffic in such a way as to deconflict the taxi plans while optimizing delay, total taxi-time, or some other airport efficiency metric. Certain input parameters related to resource demand, such as the expected landing times and the expected pushback times, are rather difficult to predict accurately. Due to uncertainty in the input data driving the taxi-planning process, the taxi-planning tool is designed such that it produces solutions that are robust to uncertainty. The taxi-planning concept presented herein, which is based on mixed-integer linear programming, is designed such that it is able to adapt to perturbations in these input conditions, as well as to account for failure in the actual execution of surface trajectories. The capabilities of the tool are illustrated in a simple hypothetical airport.
\end{abstract}

Copyright (C) 2008 P. C. Roling and H. G. Visser. This is an open access article distributed under the Creative Commons Attribution License, which permits unrestricted use, distribution, and reproduction in any medium, provided the original work is properly cited.

\section{INTRODUCTION}

The ability of the air transport system to accommodate anticipated future growth in traffic demand depends, to a significant extent, on the available capacity of the airport infrastructure. In today's air transport system, many major airports are already operating close their maximum throughput capacity. The consequence of this is that a large number of flights are delayed, which in many cases is due to congestion on the airport surface. To enlarge airport capacity, runway and taxiway systems are being expanded. However, as the density and complexity of airport of surface operations increases, safety concerns related to operations on the surface are mounting, especially under the condition of low visibility. Indeed, in the current air traffic control system, aircraft cannot be sufficiently monitored and guided under lowvisibility conditions and, as a consequence, the theoretically available capacity of an airport cannot effectively be utilized.

In response to these problems, significant research is being conducted to develop systems to enhance airport ground movement efficiency while maintaining a high level of safety in all weather conditions. In this paper, we describe a research effort pertaining to the development of a surface traffic automation system that will help controllers to better coordinate surface traffic movements related to arrival and departure traffic. More specifically, we describe the concept for a taxi-planning support tool that aims to optimize the sequencing and scheduling of traffic, such as to minimize delays and to reduce the number of stops during taxiing.

Using the envisioned taxi-planning tool, the movement of ground traffic can be planned in a conflict-free fashion, given the constraints of limited available taxiway resources. The taxi planning involves the management of arrival traffic on the taxiway system from landing runway to the apron, as well as the management of departure traffic from pushback to take-off. The proposed time-based taxi-planning concept heavily relies on the assumption that, in the near future, advanced guidance and control systems will become available that will provide aircraft with high-precision taxi capability. This capability will enable aircraft to precisely follow the deconflicted movement plans produced by the taxi-planning tool. Recent research [1] has shown that the development of such advanced guidance and control concepts is both desirable and feasible. 
The taxi-planning support tool that we present is based on a graph-theoretic airport-layout model to facilitate conflict-free aircraft movement planning. In this approach, the taxiway system is modeled as a set of nodes and links. For this purpose, taxiway intersections, runway crossings, runway and apron entries, and exits and divisions for separation constraints are defined as nodes. The aircraft movements through the taxiway system connecting the various runways with the apron are captured through a space/time network representation in which the occupancy of the nodes and links of the graph changes in time. The taxi-planning problem involves creating a time-based movement plan for each aircraft that is guaranteed to be conflict-free and takes account of both upstream and downstream requirements. Conflict-free planning implies that a specified level of separation between each aircraft is assured in the movement plans. Downstream constraints may include maintaining a certain departure sequence or meeting specified departure slot times. The capability to deal with (often hard to predict) changes in scheduled pushback times is a typical example of an upstream requirement. In addition to satisfying the above constraints, the taxi plans also aim to optimize a global (collective) performance metric that is directly related to the efficiency of taxi operations. A typical example of such a collective objective is the total taxi time. In our research, a range of collective criteria has been explored and in the planning tool a graphical user interface is provided that allows controllers to select and adjust these criteria to meet their specific needs.

Several of the explored performance criteria are based on the objective to deviate as little as possible from the "ideal" taxi plans. An ideal taxi plan is the preferred taxi plan for each individual surface movement in the absence of any other traffic. In other words, ideal taxi plans involve uncoordinated trajectories that may well contain multiple conflicts. Using the initial set of ideal taxi movement plans as its major input data set, the taxi-planning tool produces optimal conflict resolutions that result in minimal deviation from the ideal surface operations from a collective perspective. To resolve such conflicts, the taxi-planning tool utilizes two management instruments, namely, rerouting of aircraft and holding the aircraft for a certain amount of time at the apron and at various other predetermined locations on the surface.

Due to uncertainty in the input data driving the taxi planning process, the taxi-planning tool must be designed such that it produces solutions that are robust to uncertainty. Certain input parameters related to resource demand, such as the expected landing time, and particularly the expected pushback time, are rather difficult to accurately predict prior to these events. A taxi-planning tool must therefore be able to adapt to perturbations in these input conditions, as well as to account for failure in the actual execution of surface trajectories (e.g., a missed runway exit).

The online planning tool that we envision bases its activities primarily on observations of the current state of the system and on the traffic anticipated to be using the taxiway system within the planning horizon. At each planning update (a multiple of the time increment step size, typically a factor of six), the actual position on the surface is used to revise the initial conditions in the planning process. Also any revision in the estimated pushback or landing time is taken into account. The envisioned taxi-planning tool performs time/space deconfliction over a fixed planning horizon. Obviously, it is not desired that the entire surface operation is completely reshuffled every time a planning update is made. For this reason, also a freezing horizon has been introduced into the planning system. If a departing aircraft has its earliest possible pushback time before the freezing horizon, the route of that aircraft is fixed and no holding is allowed before that horizon. In the next section, we will primarily focus on the models and results for a single planning update, without considering a freezing horizon. The numerical example, however, will show the results of a calculation with multiple planning updates.

To handle the online planning problem outlined above, one of the most commonly employed operations research methods for large-scale problems has been successfully used, namely, mixed-integer linear programming (MILP) [2]. More specifically, a commercial MILP package called CPLEX has been employed [3].

In the literature, various models and algorithms to deal with the taxi movement planning problem have been explored. In [4], Smeltink et al. present a study which is in some ways very similar to the work presented herein, as it also uses an MILP formulation, employs a network based on nodes, and features a sliding window for replanning. A major difference between their work and the work presented here is that they use a sequencing-based separation, where only nodes are taken into account, separation on links is taken into account indirectly, and dummy aircraft are used to fill up gaps. While their method allows for a range of speeds, instead of a few discrete values as in the method prescribed here, it does not permit holding and rerouting of aircraft. Moreover since in their model, time is not discretized, their approach is not likely to permit planning updates at fixed time intervals.

The research by Pitfield et al. [5] relies on a Monte Carlo simulation to study potentially conflicting ground movements, including towed aircraft, at congested taxiway systems. In their simulation approach, they do not optimize and only solve conflicts by holding aircraft at certain holding points, rather than through rerouting. Gotteland et al. [6] use a genetic algorithm to optimize ground traffic. Also their model does not allow for rerouting of traffic and only considers a single taxi speed. In addition, the genetic optimization approach taken by the authors appears to be better suited for offline applications. Marín [7] defines the taxi planning model as a linear multicommodity flow network, with additional side constraints. In contrast to the model considered herein, routes between origin and destination nodes are fixed and a priori determined using a shortest path algorithm in [7]. Also, in [7], only a single performance criterion is considered, namely, the total routing time for all flights only.

This paper is organized as follows. In Section 2, the time-based surface traffic movement planning concept is introduced. Section 3 outlines the mathematical model for a single-planning update. In Section 4, the taxi-planning concept is demonstrated in a numerical example involving 


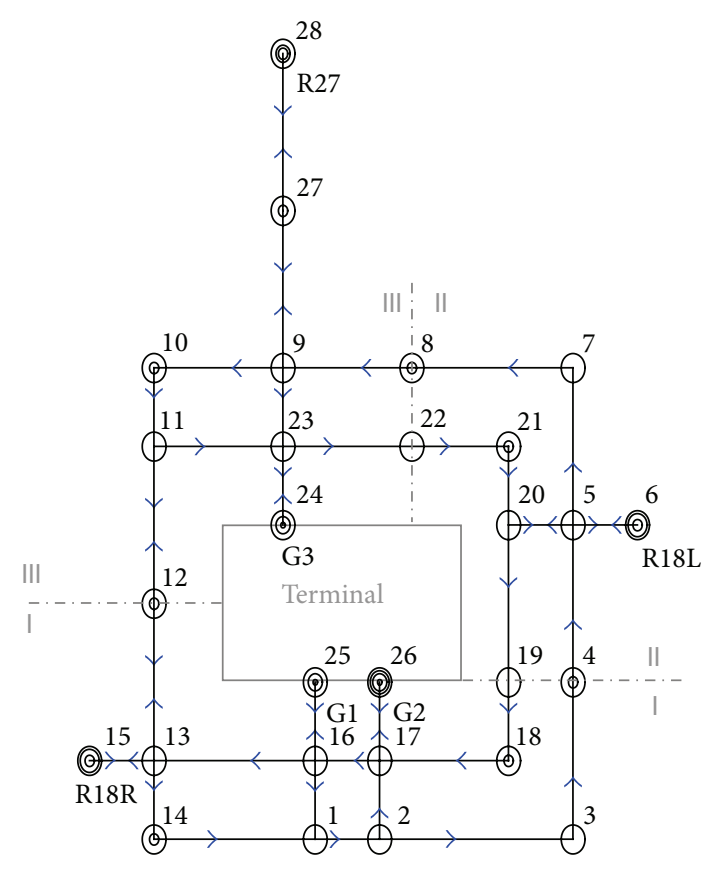

FIGURE 1: Example airport divided into sectors and elements.

a hypothetical airport. Finally, in the conclusions, the contributions of the present study are summarized, and recommendations for further research are provided.

\section{CONCEPT DESCRIPTION}

In the envisioned concept, the taxi-planning tool involves creating a (time-based) surface movement plan for each aircraft by deconflicting the uncoordinated taxi movement plans, while simultaneously optimizing a collective performance goal. The output of the tool is an optimized taxi plan that specifies for each aircraft the route to be followed and the time of arrival at each node in the selected route.

To enable the optimization of the simultaneous actions of all aircraft, a deterministic model has been formulated that involves a discretization in both time (multiperiod formulation) and space (multielement).

It is assumed that at the beginning of each planning update, a complete set of scheduled taxi movements is available for the planning interval $\left[t_{0}, t_{0}+T\right]$, including the following:

(i) the estimated landing times of the arriving aircraft;

(ii) the earliest possible pushback times for a departing aircraft;

(iii) the scheduled runway arrival times for a departing aircraft;

(iv) the initial node (apron exit node for a departing aircraft and expected runway exit node for an arriving aircraft);

(v) the final node (runway entrance node for a departing aircraft and apron entrance node for an arriving aircraft).
Note that $T$ represents the planning horizon. Since the above set pertains to individual taxi movements that are essentially uncoordinated, numerous conflicts in the trajectories may occur. Surface conflicts of various types can be distinguished, including trailing aircraft that infringes the separation requirements by closing in on the leading aircraft due to a higher taxi speed, aircraft are on an intersection of two taxiways at the same time, and two aircraft that taxi on the same taxiway in opposite directions.

The ability to provide optimal time/space deconfliction for all taxi trajectories simultaneously represents one of the major design requirements for the taxi-plannin system. The remaining requirements on which the current design has been based are as follows:

(1) Minimize a collective performance criterion that is a weighted combination of the following metrics:

(a) the total taxi time;

(b) the total holding time.

(2) Each aircraft must have its own time-based taxi movement plan.

(3) It should be possible to accommodate both one-way and two-way taxiways.

(4) It should be possible to specify a constant average taxi speed for each individual flight.

(5) The calculation time for a planning update has to be sufficiently fast to allow compatibility with the planning update rate.

To implement the above requirements, a discrete timespace network representation has been developed for the taxi-planning system. The employed time-space network representation was originally conceived in [8]. Time is treated as discrete by dividing the considered planning interval $\left[t_{0}, t_{0}+T\right]$ into periods $k$ of equal length, $k=$ $1,2, \ldots, K$, where $K$ is the total number of such periods. With respect to geographic partitioning of the prototype model, an airport is divided into several sectors, which are in turn subdivided into several elements.

In the model, the primary elements are nodes. Nodes can either be intersections, holding points or just subdivisions of a taxiway. Links connect the nodes to each other in one or both directions.

Figure 1 shows the geographic partitioning for a hypothetical example airport, featuring three runways $(27,18 \mathrm{~L}$, 18R). The aircraft holding points (or nodes) are located at the borders of the three sectors (I, II, III) shown in Figure 1.

A taxi route is split up into several taxi segments with each segment located in only one airport sector. As an example, we consider an aircraft that has to taxi from the apron exit G1 (node 25) to the departure runway 18L (node 6 ). The shortest possible taxi route, as shown in Figure 2, is

$$
\begin{aligned}
\mathrm{G} 1(\mathrm{~N} 25) & \longrightarrow \mathrm{N} 16 \longrightarrow \mathrm{N} 1 \longrightarrow \mathrm{N} 2 \longrightarrow \mathrm{N} 3 \longrightarrow \mathrm{N} 4 \longrightarrow \mathrm{N} 5 \\
& \longrightarrow \mathrm{R} 18 \mathrm{~L}(\mathrm{~N} 6) .
\end{aligned}
$$




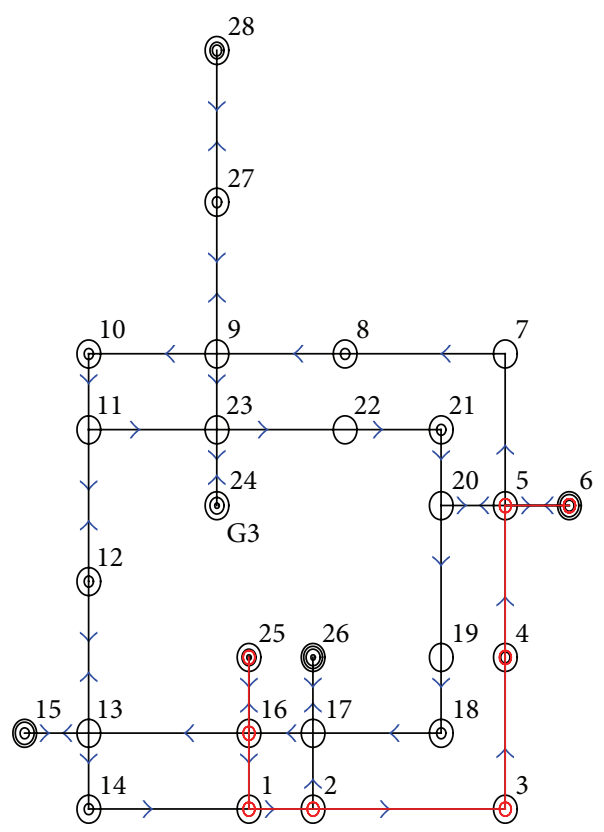

FIgURE 2: Taxi route from Gate 1 to Runway 18L.

In this notation, $\mathrm{N} x$ stands for node $x, \mathrm{G} x$ for the node corresponding to apron exit (Gate) $x$, and $\mathrm{R} x$ for the node belonging to runway $x$. This taxi route is split up in two taxi segments. The first taxi segment (which falls in sector I) is

$$
\mathrm{G} 1(\mathrm{~N} 25) \longrightarrow \mathrm{N} 16 \longrightarrow \mathrm{N} 1 \longrightarrow \mathrm{N} 2 \longrightarrow \mathrm{N} 3 \longrightarrow \mathrm{N} 4 \text {. }
$$

The second segment (which falls in sector II) is

$$
\mathrm{N} 4 \longrightarrow \mathrm{N} 5 \longrightarrow \mathrm{R} 18 \mathrm{~L}(\mathrm{~N} 6) \text {. }
$$

The maximum number of segments is dependant on the taxi route because not every taxi route passes through the same number of sectors. Since one of the requirements is that an aircraft can be rerouted, a number of alternative taxi routes are assigned to each aircraft in addition to the easiest route. The easiest route is the route that passes the least number of nodes and is usually also the shortest.

Since the performance index is based on a group (collective) criterion, there is a danger that certain individual taxi movements are penalized more than others in terms of the delay that they have to incur. To limit the calculation time and avoid grossly inequitable departure delays for individual aircraft, a maximum amount of delay is specified by the user.

\section{TAXI PLANNING MODEL}

In this section, the MILP model for the taxi-planning problem is defined, based on the time-space network representation described in the previous section.

\subsection{Decision variables}

The variables that are used to define the taxi planning problem are binary in nature as follows. $x_{f, d, r, s}$. The indices indicate a segment $(s)$ of route $(r)$ belonging to flight $(f)$ with a certain seconds of delay $(d)$. Thus, $x_{f, d, r, s}$ is 1 if flight $(f)$ takes route $(r)$ and has delay $(d)$ in segment $(s)$, and is 0 otherwise.

$w_{f, d, r, s}$. If a flight $(f)$ with seconds of delay $(d)$ waits for one period on a node at the start of segment $(s)$ of route $(r)$, then $w_{f, d, r, s}$ is 1 , and 0 otherwise. This variable is only used for blocking a node during the time the aircraft is waiting on it.

\subsection{Objective function}

The objective function $(Z)$ considered in this study is a weighted combination of the total taxi time and the total holding time:

$$
Z=\sum_{f=1}^{f_{\max }} \sum_{r=1}^{r_{\max (f)}} \sum_{d=0}^{d_{\max }} K_{f}\left[C_{r}+C_{d}\right] x_{f, d, r, s_{\max (f, r)}}
$$

where $K_{f}$ is the flight priority cost factor. Specifying a higher value of $K_{f}$ for a given flight $(f)$ relative to the other flights makes delay for that particular flight relatively more expensive, causing the optimization process to avoid adding delay to that flight.

The route cost $C_{r}$ is equal to the route time cost times the total active taxi time (thus without delay):

$$
C_{r}=K_{r}\left(T_{\text {start }(f, r)}-T_{\text {end }(f, r)}\right),
$$

where $K_{r}$ is the user-specified route time cost factor; $T_{\text {start }(f, r)}$ is the time on which route $(r)$ of flight $(f)$ starts; $T_{\operatorname{end}(f, r)}$ is the time on which route $(r)$ of flight $(f)$ ends.

The delay cost $C_{d}$ specified in the objective function is defined as

$$
C_{d}=K_{d} d
$$

\subsection{Constraints}

The discrete model developed is used in conjunction with a mixed-integer linear programming (MILP) approach. The linear constraints that are used this model are as follows.

(i) Node occupancy is a holding constraint that is needed to avoid conflicts by limiting the occupation of a node to a single flight at a time:

$$
\begin{aligned}
& \mathrm{NO}_{t, n}: \sum_{f=1}^{f_{\max }} \sum_{r=1}^{r_{\max (f)}} \sum_{s=1}^{s_{\max (f, r)}} \sum_{d=0}^{d_{\max (f, r, s)}} n_{f, r, s, n, t-d} x_{f, d, r, s} \leq 1 \\
& \forall t=t_{\min }, \ldots, t_{\max }, \\
& \forall n=1, \ldots, n_{\text {tot }}, n \neq n_{\text {hold }()} \text {, }
\end{aligned}
$$

where $n_{f, r, s, n, t-d}$ is a binary constant that indicates if segment $(s)$ of route $(r)$ belonging to flight $(f)$ occupies node $(n)$ if it has an amount of delay $(d)$ at time $(t)$. 
(ii) Link occupancy is a separation constraint and is similar to the node occupancy constraint as link occupancy limits the occupation of a link to a single flight at a time:

$$
\begin{aligned}
& \mathrm{LO}_{l, n}: \sum_{f=1}^{f_{\max }} \sum_{r=1}^{r_{\max (f)}} \sum_{s=1}^{s_{\max (f, r)}} \sum_{d=0}^{d_{\max (f, r, s)}} l_{f, r, s, l, t-d} x_{f, d, r, s} \leq 1 \\
& \forall t=t_{\min }, \ldots, t_{\max }, \\
& \forall l=1, \ldots, l_{\mathrm{tot}},
\end{aligned}
$$

where $l_{f, r, s, l, t-d}$ is a binary constant that indicates if a flight segment $(s)$ of route $(r)$ belonging to flight $(f)$ occupies link $(l)$ if it has an amount of delay $(d)$ at time $(t)$.

(iii) Route and delay choice is a flow conservation constraint that forces a route and delay to be chosen for every flight and segment, even if it is an empty segment in which the flight has already finished its route:

$$
\begin{array}{r}
\operatorname{RADC}_{f, s}: \sum_{r=1}^{r_{\max (f)} d_{\max (f, r, s)}} x_{d=0} x_{f, d, r, s}=1 \\
\forall f=1, \ldots, f_{\max }, \\
\forall s=1, \ldots, s_{\max (f) .}
\end{array}
$$

(iv) Waiting times is a sequence and flow conservation constraint and ties all segments and waiting periods together, where $\Delta t$ is the time increment step size and is equal to one period. In the example scenarios, the value of $\Delta t=10$ seconds

$$
\begin{array}{r}
W T_{f, r, s}: \sum_{d=0}^{d_{\max (f, r, s)}} 2^{d / \Delta t} x_{f, d, r, s}-2^{d / \Delta t} x_{f, d, r, s-1}-2^{d / \Delta t} w_{f, d, r, s}=0 \\
\forall f=1, \ldots, f_{\text {tot }}, \\
\forall r=1, \ldots, r_{\text {tot }(f),} \\
\forall s=2, \ldots, s_{\text {tot }(f, r) .}
\end{array}
$$

If extra delay is "inserted" between two segments, this constraint forces the time gap between these segments to be filled with waiting points. For example, if delay is zero for segment one and twenty seconds for segment two, the summation-if zero values of $x$ are omitted-reduces to the following equation:

$$
\begin{gathered}
2^{2} x_{f, 20, r, 2}-2^{0} x_{f, 0, r, 1}-2^{0} w_{f, 0, r, 2}-2^{1} w_{f, 10, r, 2}-2^{2} w_{f, 20, r, 2}=0 \\
\longrightarrow 4-1-w_{f, 0, r, 2}-2 w_{f, 10, r, 2}-4 w_{f, 20, r, 2}=0 .
\end{gathered}
$$

The above equality can only be satisfied if $w_{f, 0, r, 2}=1$, $w_{f, 10, r, 2}=1$, and $w_{f, 20, r, 2}=0$.

\section{NUMERICAL EXAMPLE}

To illustrate the developed concept, a numerical example is presented related to the hypothetical airport shown in Figure 1. The major characteristics of this airport are as follows:

(a) it has an outer and an inner taxiway, which are oneway taxiways;

(b) it has a two-way taxiway on which aircraft can taxi in both directions;

(c) the apron lies in the center of the taxiway system, so aircraft can taxi clockwise or counterclockwise from the starting node (either the landing runway or the apron exit) to the end node (either the apron entrance or takeoff runway);

(d) there are multiple apron exits and entrances;

(e) the airport-use configuration involves three runways that are all operated in a segregated mode.

Aircraft entering the taxiway system are divided into fast and slow aircraft. Fast aircraft taxi with an average speed of $16 \mathrm{~m} / \mathrm{s}$, while slow aircraft taxi with an average speed of $8 \mathrm{~m} / \mathrm{s}$. In the numerical example, the safe separation distance $\mathrm{S}_{\text {eparation }}$ is set at $200 \mathrm{~m}$. Based on this separation distance, the line elements (links) are configured as shown in Table 1.

With the lengths of the line elements, the taxi-speed options, and a period length of 10 seconds, the number of periods needed to cover each line element for a given taxi speed can be approximately determined.

Let us, for example, consider line element connecting Node 2 and Node 3 with a length of $300 \mathrm{~m}$. It takes 37.5 seconds or 4 time periods to cover the $300 \mathrm{~m}$ distance with a taxi speed of $8 \mathrm{~m} / \mathrm{s}$. In the last period, Node 3 is also occupied. The resulting set of the number of time periods needed is included in Table 1. It should be noted that it is possible for an aircraft to block multiple nodes and links during the same period, so the rounding of time to periods does not accumulate during the route.

To test the taxi-planning concept for the hypothetic airport, a scenario has been created that involves the movement planning of eight aircraft (six departing and two arriving) using a planning horizon $T$ of 42 periods. In view of the assumed period length of 10 seconds, the planning interval consists of 420 seconds or (6 minutes).

As mentioned earlier, a set of the ideal uncoordinated movement plans is calculated at the start of the planning update. Table 2 lists the aircraft planning set for the present example scenario. It should be noted that this planning set is purposely complex to give a good indication of the conflicts to be solved.

With the information provided in Tables 1 and 2, the ideal taxi plan for each aircraft is determined. These individually ideal taxi plans are shown in Table 3 . The bold printed time in Table 3 indicates the applied planning horizon. The use of a planning horizon helps to reduce the complexity of the optimization problem. Note that the pushback time is specified in such a way that departures can not only be delayed, but can also be scheduled earlier due to the use of longer routes.

The bold numbers in Table 3 indicate the various conflicts. If aircraft would indeed taxi according to the ideal taxi plan, two kinds of conflicts can be observed as follows. 
TABLE 1: The line element lengths and the number of periods needed to cover the elements with different taxi speeds.

\begin{tabular}{|c|c|c|c|c|}
\hline From node & To node & Distance (meters) & Periods at $16 \mathrm{~m} / \mathrm{s}$ & Periods at $8 \mathrm{~m} / \mathrm{s}$ \\
\hline N01 & N02 & 100 & 1 & 2 \\
\hline N01 & N14 & 250 & 2 & 4 \\
\hline N01 & N16 & 100 & 1 & 2 \\
\hline N02 & N03 & 300 & 2 & 4 \\
\hline N02 & N17 & 100 & 1 & 2 \\
\hline N03 & N04 & 200 & 2 & 3 \\
\hline N04 & N05 & 200 & 2 & 3 \\
\hline N05 & N06 & 100 & 1 & 2 \\
\hline N05 & N20 & 100 & 1 & 2 \\
\hline N05 & N07 & 200 & 2 & 3 \\
\hline N07 & N08 & 250 & 2 & 4 \\
\hline N08 & N09 & 200 & 2 & 3 \\
\hline N09 & N23 & 100 & 1 & 2 \\
\hline N09 & $\mathrm{N} 27$ & 200 & 2 & 3 \\
\hline N09 & N10 & 200 & 2 & 3 \\
\hline N10 & N11 & 100 & 1 & 2 \\
\hline N11 & N12 & 200 & 2 & 3 \\
\hline N11 & N23 & 200 & 2 & 3 \\
\hline N12 & N13 & 200 & 2 & 3 \\
\hline N13 & N15 & 100 & 1 & 2 \\
\hline N13 & N16 & 250 & 2 & 4 \\
\hline N13 & N14 & 100 & 1 & 2 \\
\hline N16 & N17 & 100 & 1 & 2 \\
\hline N16 & N25 & 100 & 1 & 2 \\
\hline N17 & N26 & 100 & 1 & 2 \\
\hline N17 & N18 & 200 & 2 & 3 \\
\hline N18 & N19 & 100 & 1 & 2 \\
\hline N19 & N20 & 200 & 2 & 3 \\
\hline N20 & N21 & 100 & 1 & 2 \\
\hline $\mathrm{N} 21$ & N22 & 150 & 1 & 2 \\
\hline $\mathrm{N} 22$ & N23 & 200 & 2 & 3 \\
\hline N23 & N24 & 100 & 1 & 2 \\
\hline $\mathrm{N} 27$ & N28 & 200 & 2 & 3 \\
\hline
\end{tabular}

TABLE 2: List with the departing and arriving aircraft, the taxi speed, the starting point (departure gate or arrival runway), the destination point (arrival gate or departure runway), the start time (earliest pushback time or runway arrival time), and the destination time (latest gate arrival time or allocated runway departure time).

\begin{tabular}{|c|c|c|c|c|c|}
\hline Aircraft & Start & Time & Destination & Time & Speed $(\mathrm{m} / \mathrm{s})$ \\
\hline 1 (departing) & G2 (N26) & 0:01:10 & R18R (N15) & 0:06:00 & 8 \\
\hline 2 (departing) & G3 (N24) & 0:01:00 & R18R (N15) & 0:07:00 & 16 \\
\hline 3 (departing) & G1 (N25) & $0: 01: 40$ & R18L (N06) & 0:07:00 & 16 \\
\hline 4 (departing) & G1 (N25) & 0:01:20 & R18L (N06) & 0:07:30 & 8 \\
\hline 5 (departing) & G1 (N25) & 0:02:40 & R18L (N06) & 0:08:30 & 16 \\
\hline 6 (departing) & G3 (N24) & 0:02:20 & R18L (N06) & 0:08:30 & 8 \\
\hline 7 (arriving) & R27 (N28) & 0:00:00 & G2 (N26) & 0:09:30 & 8 \\
\hline 8 (arriving) & R27 (N28) & 0:00:30 & G2 (N26) & 0:09:00 & 8 \\
\hline
\end{tabular}


TABLe 3: Ideal taxi plan (with conflicts).

\begin{tabular}{|c|c|c|c|c|c|c|c|c|}
\hline \multirow{2}{*}{ Time } & \multicolumn{8}{|c|}{ Aircraft } \\
\hline & 1 & 2 & 3 & 4 & 5 & 6 & 7 & 8 \\
\hline $0: 00$ & & & & & & & 28 & \\
\hline $0: 10$ & & & & & & & $27-28$ & \\
\hline $0: 20$ & & & & & & & $27-28$ & \\
\hline $0: 30$ & & & & & & & $27-28$ & 28 \\
\hline $0: 40$ & & & & & & & $27-28$ & $27-28$ \\
\hline $0: 50$ & & & & & & & $27-28$ & $27-28$ \\
\hline $1: 00$ & & & & & & & $27-28$ & $27-28$ \\
\hline $1: 10$ & & & & & & & $9-27$ & $27-28$ \\
\hline $1: 20$ & & & & & & & $9-27$ & $27-28$ \\
\hline $1: 30$ & & & & & & & $9-27$ & $27-28$ \\
\hline $1: 40$ & & & & & & & $9-27$ & $9-27$ \\
\hline $1: 50$ & & & & & & & $9-27$ & $9-27$ \\
\hline $2: 00$ & & & & 25 & & & $9-27$ & $9-27$ \\
\hline $2: 10$ & & & & $16-25$ & & & $9-23$ & $9-27$ \\
\hline $2: 20$ & & & & $16-25$ & & & $9-23$ & $9-27$ \\
\hline $2: 30$ & & & & $16-25$ & & & $9-23$ & $9-27$ \\
\hline $2: 40$ & & 24 & & $1-16$ & & & $22-23$ & $9-23$ \\
\hline $2: 50$ & & $23-24$ & & $1-16$ & & & $22-23$ & $9-23$ \\
\hline $3: 00$ & & $23-24$ & & $1-16$ & & & $22-23$ & $9-23$ \\
\hline $3: 10$ & 26 & $22-23$ & & $1-2$ & & & $22-23$ & $22-23$ \\
\hline $3: 20$ & $17-26$ & $22-23$ & & $1-2$ & & & $22-23$ & $22-23$ \\
\hline $3: 30$ & $17-26$ & $22-23$ & & $1-2$ & & & $22-23$ & $22-23$ \\
\hline $3: 40$ & $17-26$ & $21-22$ & & $2-3$ & & & $21-22$ & $22-23$ \\
\hline $3: 50$ & $16-17$ & $21-22$ & 25 & $2-3$ & & & $21-22$ & $22-23$ \\
\hline 4:00 & $16-17$ & $20-21$ & $16-25$ & $2-3$ & & & $21-22$ & $22-23$ \\
\hline $4: 10$ & $16-17$ & $20-21$ & $16-25$ & $2-3$ & & & $21-22$ & $21-22$ \\
\hline $4: 20$ & $13-16$ & $19-20$ & $1-16$ & $2-3$ & & & $20-21$ & $21-22$ \\
\hline $4: 30$ & $13-16$ & $19-20$ & $1-16$ & $2-3$ & & & $20-21$ & $21-22$ \\
\hline $4: 40$ & $13-16$ & $19-20$ & $1-2$ & $2-3$ & & 24 & $20-21$ & $21-22$ \\
\hline $4: 50$ & $13-16$ & $18-19$ & $1-2$ & $2-3$ & & $23-24$ & $19-20$ & $20-21$ \\
\hline $5: 00$ & $13-16$ & $18-19$ & $2-3$ & $3-4$ & & $23-24$ & $19-20$ & $20-21$ \\
\hline $5: 10$ & $13-16$ & $17-18$ & $2-3$ & $3-4$ & & $23-24$ & $19-20$ & $20-21$ \\
\hline $5: 20$ & $13-16$ & $17-18$ & $2-3$ & $3-4$ & 25 & $22-23$ & $19-20$ & $19-20$ \\
\hline $5: 30$ & $13-15$ & $17-18$ & $2-3$ & $3-4$ & $16-25$ & $22-23$ & $19-20$ & $19-20$ \\
\hline $5: 40$ & $13-15$ & $16-17$ & $3-4$ & $3-4$ & $16-25$ & $22-23$ & $19-20$ & $19-20$ \\
\hline $5: 50$ & $13-15$ & $16-17$ & $3-4$ & $3-4$ & $1-16$ & $22-23$ & $18-19$ & $19-20$ \\
\hline $6: 00$ & & $13-16$ & $3-4$ & $4-5$ & $1-16$ & $22-23$ & $18-19$ & $19-20$ \\
\hline $6: 10$ & & $13-16$ & 4-5 & 4-5 & $1-2$ & $22-23$ & $18-19$ & $19-20$ \\
\hline $6: 20$ & & $13-16$ & $4-5$ & $4-5$ & $1-2$ & $21-22$ & $17-18$ & 18-19 \\
\hline $6: 30$ & & $13-16$ & 4-5 & 4-5 & $2-3$ & $21-22$ & $17-18$ & $18-19$ \\
\hline $6: 40$ & & $13-15$ & $5-6$ & 4-5 & $2-3$ & $21-22$ & $17-18$ & 18-19 \\
\hline $6: 50$ & & $13-15$ & $5-6$ & $4-5$ & $2-3$ & $21-22$ & $17-18$ & $17-18$ \\
\hline 7:00 & & & & $5-6$ & $2-3$ & $20-21$ & $17-18$ & $17-18$ \\
\hline $7: 10$ & & & & $5-6$ & $3-4$ & $20-21$ & $17-18$ & $17-18$ \\
\hline $7: 20$ & & & & $5-6$ & $3-4$ & $20-21$ & $17-26$ & $17-18$ \\
\hline $7: 30$ & & & & & $3-4$ & $5-20$ & $17-26$ & $17-18$ \\
\hline $7: 40$ & & & & & $4-5$ & $5-20$ & $17-26$ & $17-18$ \\
\hline $7: 50$ & & & & & 4-5 & $5-20$ & & $17-26$ \\
\hline 8:00 & & & & & $4-5$ & $5-6$ & & $17-26$ \\
\hline $8: 10$ & & & & & $5-6$ & $5-6$ & & $17-26$ \\
\hline $8: 20$ & & & & & $5-6$ & $5-6$ & & \\
\hline
\end{tabular}




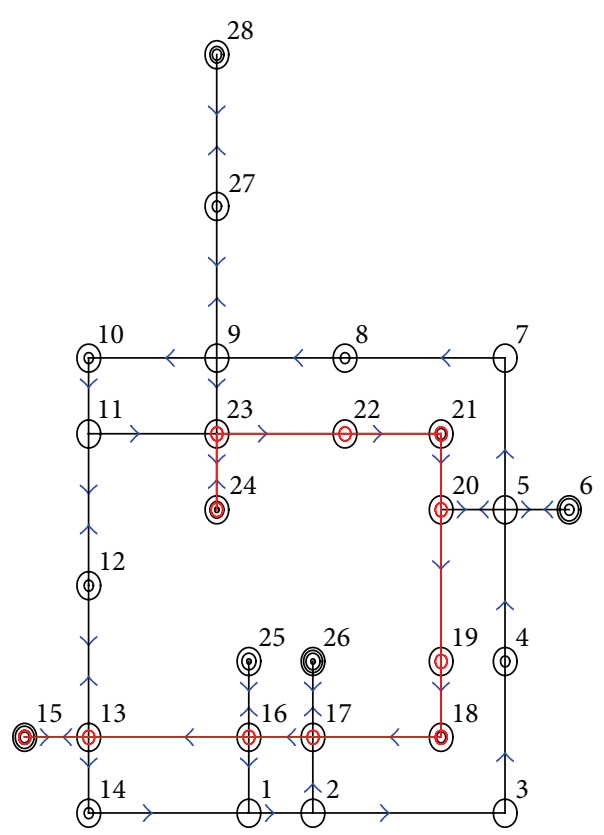

Figure 3: Aircraft 2, shortest route.

(1) Two aircraft are at an intersection at the same time. According to the individually ideal plans, aircraft 1 and 3 both cross node 16 at time 4:10.

(2) Two aircraft close in while taxiing on the same link. If the ideal plans were to be followed, aircraft 3 would overtake aircraft 4 , and aircraft 7 would violate the separation with aircraft 8 .

To resolve the conflicts and optimize the traffic flow, a performance index has to be specified. At present, two different criteria as well as any weighted combination of these criteria could be selected. In the example presented herein, the primary objective is to minimize the taxi travel time while penalizing delay twice as heavy.

The results for this particular scenario are shown in Table 4. In Table 4, the optimized and deconflicted taxi plans are shown for the 8 aircraft moving on the surface. The bold printed cells indicate changes and arrows indicate delayed pushback.

In the optimal solution, the following features can be observed.

(1) Aircraft 1 taxies according to its ideal taxi plan.

(2) Aircraft 2 follows a longer route than the shortest one, as is illustrated in Figures 3 and 4, and to arrive at the takeoff runway on the stated time, it departs the apron earlier. This is still, however, later than the earliest pushback time stated in Table 2 .

(3) Aircraft 3 follows a longer route, as aircraft 2 does. It therefore also leaves the apron earlier to be at the departure runway on time.

(4) Aircraft 4 taxies according to its ideal taxi plan.

(5) Aircraft 5 taxies according to its ideal taxi plan.

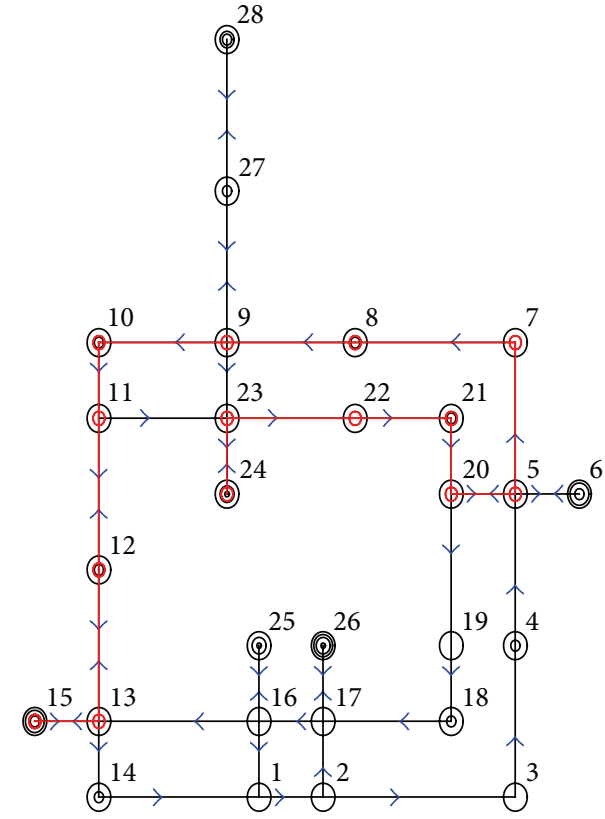

FIgURE 4: Aircraft 2, rerouted.

(6) Aircraft 6 follows the shortest route, but is delayed at the gate for two periods and therefore arrives at the runway 20 seconds later.

(7) Aircraft 7 taxies according to its ideal taxi plan.

(8) Aircraft 8 follows the shortest route, but holds at the runway exit for three periods and therefore arrives at the gate 30 seconds later.

Due to that fact that planning is restricted to within a fixed horizon, a conflict may still exist beyond the planning horizon. Table 4 shows such a conflict for aircraft 5 and 6 at time 8:20. In the planning update 2 minutes later, shown in Table 5, this conflict is resolved by delaying flight 6 on node 21 for one period. The rest of the planning remains unchanged.

The computational burden for a problem of the complexity as presented herein is modest, even though the situation shown in the example is purposely overcomplex. On a standard PC, the calculation of a full run with eight aircraft and many conflicts takes about 5 seconds, with planning updates running significantly faster due to the reduced number of conflicts. It should be noted that the computational time does significantly increase with an increase of the number of flights. For example, doubling the number of flights in the numerical example from 8 to 16 causes the calculation time to go up from 5 to about 20 seconds. It is therefore important that both the airport model and the flight schedule are well structured and that the planning horizon is kept within reasonable limits to ensure that the MILP problem remains computationally tractable. Preliminary runs with a full scale flight schedule, with 20 aircraft divided regularly over 15 minutes, show that, due to the reduced number of conflicts and thus complexity, the computational time remains within a couple of seconds. 
TABLE 4: First planning update (conflict after horizon).

\begin{tabular}{|c|c|c|c|c|c|c|c|c|}
\hline \multirow{2}{*}{ Time } & \multicolumn{8}{|c|}{ Aircraft } \\
\hline & 1 & 2 & 3 & 4 & 5 & 6 & 7 & 8 \\
\hline $0: 00$ & & & & & & & 28 & \\
\hline $0: 10$ & & & & & & & $27-28$ & \\
\hline $0: 20$ & & & & & & & $27-28$ & \\
\hline $0: 30$ & & & & & & & $27-28$ & $\Downarrow$ \\
\hline $0: 40$ & & & & & & & $27-28$ & $\Downarrow$ \\
\hline $0: 50$ & & & & & & & $27-28$ & $\Downarrow$ \\
\hline $1: 00$ & & & & & & & $27-28$ & 28 \\
\hline $1: 10$ & & 24 & & & & & $9-27$ & $27-28$ \\
\hline $1: 20$ & & $23-24$ & & & & & $9-27$ & $27-28$ \\
\hline $1: 30$ & & $23-24$ & & & & & $9-27$ & $27-28$ \\
\hline $1: 40$ & & $22-23$ & & & & & $9-27$ & $27-28$ \\
\hline $1: 50$ & & $22-23$ & & & & & $9-27$ & $27-28$ \\
\hline $2: 00$ & & $22-23$ & & 25 & & & $9-27$ & $27-28$ \\
\hline $2: 10$ & & $21-22$ & & $16-25$ & & & $9-23$ & $9-27$ \\
\hline $2: 20$ & & $21-22$ & & $16-25$ & & & $9-23$ & $9-27$ \\
\hline $2: 30$ & & $20-21$ & 25 & $16-25$ & & & $9-23$ & $9-27$ \\
\hline $2: 40$ & & $20-21$ & $16-25$ & $1-16$ & & & $22-23$ & $9-27$ \\
\hline $2: 50$ & & $5-20$ & $16-25$ & $1-16$ & & & $22-23$ & $9-27$ \\
\hline $3: 00$ & & $5-20$ & $13-16$ & $1-16$ & & & $22-23$ & $9-27$ \\
\hline $3: 10$ & 26 & $5-7$ & $13-16$ & $1-2$ & & & $22-23$ & $9-23$ \\
\hline $3: 20$ & $17-26$ & $5-7$ & $13-16$ & $1-2$ & & & $22-23$ & $9-23$ \\
\hline $3: 30$ & $17-26$ & $5-7$ & $13-16$ & $1-2$ & & & $22-23$ & $9-23$ \\
\hline $3: 40$ & $17-26$ & $7-8$ & $12-13$ & $2-3$ & & & $21-22$ & $22-23$ \\
\hline $3: 50$ & $16-17$ & $7-8$ & $12-13$ & $2-3$ & & & $21-22$ & $22-23$ \\
\hline 4:00 & $16-17$ & $7-8$ & $12-13$ & $2-3$ & & & $21-22$ & $22-23$ \\
\hline $4: 10$ & $16-17$ & $7-8$ & $11-12$ & $2-3$ & & & $21-22$ & $22-23$ \\
\hline $4: 20$ & $13-16$ & $8-9$ & $11-12$ & $2-3$ & & & $20-21$ & $22-23$ \\
\hline $4: 30$ & $13-16$ & $8-9$ & $11-12$ & $2-3$ & & & $20-21$ & $22-23$ \\
\hline $4: 40$ & $13-16$ & 8-9 & $11-23$ & $2-3$ & & $\Downarrow$ & $20-21$ & $21-22$ \\
\hline $4: 50$ & $13-16$ & $9-10$ & $11-23$ & $2-3$ & & $\Downarrow$ & $19-20$ & $21-22$ \\
\hline $5: 00$ & $13-16$ & $9-10$ & $11-23$ & $3-4$ & & 24 & $19-20$ & $21-22$ \\
\hline $5: 10$ & $13-16$ & $9-10$ & $22-23$ & $3-4$ & & $23-24$ & $19-20$ & $21-22$ \\
\hline $5: 20$ & $13-16$ & $10-11$ & $22-23$ & $3-4$ & 25 & $23-24$ & $19-20$ & $20-21$ \\
\hline $5: 30$ & $13-15$ & $10-11$ & $22-23$ & $3-4$ & $16-25$ & $23-24$ & $19-20$ & $20-21$ \\
\hline $5: 40$ & $13-15$ & $11-12$ & $21-22$ & $3-4$ & $16-25$ & $22-23$ & $19-20$ & $20-21$ \\
\hline $5: 50$ & $13-15$ & $11-12$ & $21-22$ & $3-4$ & $1-16$ & $22-23$ & $18-19$ & $19-20$ \\
\hline $6: 00$ & & $11-12$ & $20-21$ & $4-5$ & $1-16$ & $22-23$ & $18-19$ & $19-20$ \\
\hline $6: 10$ & & $12-13$ & $20-21$ & $4-5$ & $1-2$ & $22-23$ & $18-19$ & $19-20$ \\
\hline $6: 20$ & & $12-13$ & $5-20$ & $4-5$ & $1-2$ & $22-23$ & $17-18$ & $19-20$ \\
\hline $6: 30$ & & $12-13$ & $5-20$ & $4-5$ & $2-3$ & $22-23$ & $17-18$ & $19-20$ \\
\hline $6: 40$ & & $13-15$ & $5-6$ & 4-5 & $2-3$ & $21-22$ & $17-18$ & $19-20$ \\
\hline $6: 50$ & & $13-15$ & $5-6$ & $4-5$ & $2-3$ & $21-22$ & $17-18$ & $18-19$ \\
\hline 7:00 & & & & $5-6$ & $2-3$ & $21-22$ & $17-18$ & $18-19$ \\
\hline $7: 10$ & & & & $5-6$ & $3-4$ & $21-22$ & $17-18$ & 18-19 \\
\hline $7: 20$ & & & & $5-6$ & $3-4$ & $20-21$ & $17-26$ & $17-18$ \\
\hline $7: 30$ & & & & & $3-4$ & $20-21$ & $17-26$ & $17-18$ \\
\hline $7: 40$ & & & & & $4-5$ & $20-21$ & $17-26$ & $17-18$ \\
\hline $7: 50$ & & & & & $4-5$ & $5-20$ & & $17-18$ \\
\hline 8:00 & & & & & $4-5$ & $5-20$ & & $17-18$ \\
\hline $8: 10$ & & & & & $5-6$ & $5-20$ & & $17-18$ \\
\hline $8: 20$ & & & & & $5-6$ & $5-6$ & & $17-26$ \\
\hline $8: 30$ & & & & & & $5-6$ & & $17-26$ \\
\hline $8: 40$ & & & & & & $5-6$ & & $17-26$ \\
\hline
\end{tabular}


TABLE 5: Planning update after 2 minutes (conflict-free).

\begin{tabular}{|c|c|c|c|c|c|c|c|c|}
\hline \multirow{2}{*}{ Time } & \multicolumn{8}{|c|}{ Aircraft } \\
\hline & 1 & 2 & 3 & 4 & 5 & 6 & 7 & 8 \\
\hline $0: 00$ & & & & & & & 28 & \\
\hline $0: 10$ & & & & & & & $27-28$ & \\
\hline $0: 20$ & & & & & & & $27-28$ & \\
\hline $0: 30$ & & & & & & & $27-28$ & \\
\hline $0: 40$ & & & & & & & $27-28$ & \\
\hline 0:50 & & & & & & & $27-28$ & \\
\hline $1: 00$ & & & & & & & $27-28$ & 28 \\
\hline $1: 10$ & & 24 & & & & & $9-27$ & $27-28$ \\
\hline $1: 20$ & & $23-24$ & & & & & $9-27$ & $27-28$ \\
\hline 1:30 & & $23-24$ & & & & & $9-27$ & $27-28$ \\
\hline $1: 40$ & & $22-23$ & & & & & $9-27$ & $27-28$ \\
\hline $1: 50$ & & $22-23$ & & & & & $9-27$ & $27-28$ \\
\hline 2:00 & & $22-23$ & & 25 & & & $9-27$ & $27-28$ \\
\hline $2: 10$ & & $21-22$ & & $16-25$ & & & $9-23$ & $9-27$ \\
\hline $2: 20$ & & $21-22$ & & $16-25$ & & & $9-23$ & $9-27$ \\
\hline $2: 30$ & & $20-21$ & 25 & $16-25$ & & & $9-23$ & $9-27$ \\
\hline $2: 40$ & & $20-21$ & $16-25$ & $1-16$ & & & $22-23$ & $9-27$ \\
\hline $2: 50$ & & $5-20$ & $16-25$ & $1-16$ & & & $22-23$ & $9-27$ \\
\hline 3:00 & & $5-20$ & $13-16$ & $1-16$ & & & $22-23$ & $9-27$ \\
\hline $3: 10$ & 26 & $5-7$ & $13-16$ & $1-2$ & & & $22-23$ & $9-23$ \\
\hline $3: 20$ & $17-26$ & $5-7$ & $13-16$ & $1-2$ & & & $22-23$ & $9-23$ \\
\hline $3: 30$ & $17-26$ & $5-7$ & $13-16$ & $1-2$ & & & $22-23$ & $9-23$ \\
\hline 3:40 & $17-26$ & $7-8$ & $12-13$ & $2-3$ & & & $21-22$ & $22-23$ \\
\hline 3:50 & $16-17$ & $7-8$ & $12-13$ & $2-3$ & & & $21-22$ & $22-23$ \\
\hline 4:00 & $16-17$ & $7-8$ & $12-13$ & $2-3$ & & & $21-22$ & $22-23$ \\
\hline $4: 10$ & $16-17$ & $7-8$ & $11-12$ & $2-3$ & & & $21-22$ & $22-23$ \\
\hline $4: 20$ & $13-16$ & $8-9$ & $11-12$ & $2-3$ & & & $20-21$ & $22-23$ \\
\hline 4:30 & $13-16$ & $8-9$ & $11-12$ & $2-3$ & & & $20-21$ & $22-23$ \\
\hline 4:40 & $13-16$ & $8-9$ & $11-23$ & $2-3$ & & & $20-21$ & $21-22$ \\
\hline 4:50 & $13-16$ & $9-10$ & $11-23$ & $2-3$ & & & $19-20$ & $21-22$ \\
\hline $5: 00$ & $13-16$ & $9-10$ & $11-23$ & $3-4$ & & 24 & $19-20$ & $21-22$ \\
\hline $5: 10$ & $13-16$ & $9-10$ & $22-23$ & $3-4$ & & $23-24$ & $19-20$ & $21-22$ \\
\hline $5: 20$ & $13-16$ & $10-11$ & $22-23$ & $3-4$ & 25 & $23-24$ & $19-20$ & $20-21$ \\
\hline $5: 30$ & $13-15$ & $10-11$ & $22-23$ & $3-4$ & $16-25$ & $23-24$ & $19-20$ & $20-21$ \\
\hline $5: 40$ & $13-15$ & $11-12$ & $21-22$ & $3-4$ & $16-25$ & $22-23$ & $19-20$ & $20-21$ \\
\hline $5: 50$ & $13-15$ & $11-12$ & $21-22$ & $3-4$ & $1-16$ & $22-23$ & $18-19$ & $19-20$ \\
\hline 6:00 & & $11-12$ & $20-21$ & $4-5$ & $1-16$ & $22-23$ & $18-19$ & $19-20$ \\
\hline $6: 10$ & & $12-13$ & $20-21$ & $4-5$ & $1-2$ & $22-23$ & $18-19$ & $19-20$ \\
\hline $6: 20$ & & $12-13$ & $5-20$ & $4-5$ & $1-2$ & $22-23$ & $17-18$ & $19-20$ \\
\hline $6: 30$ & & $12-13$ & $5-20$ & $4-5$ & $2-3$ & $22-23$ & $17-18$ & $19-20$ \\
\hline $6: 40$ & & $13-15$ & $5-6$ & $4-5$ & $2-3$ & $21-22$ & $17-18$ & $19-20$ \\
\hline $6: 50$ & & $13-15$ & $5-6$ & $4-5$ & $2-3$ & $21-22$ & $17-18$ & 18-19 \\
\hline 7:00 & & & & $5-6$ & $2-3$ & $21-22$ & $17-18$ & $18-19$ \\
\hline 7:10 & & & & $5-6$ & $3-4$ & $21-22$ & $17-18$ & $18-19$ \\
\hline 7:20 & & & & $5-6$ & $3-4$ & 21 & $17-26$ & $17-18$ \\
\hline 7:30 & & & & & $3-4$ & $20-21$ & $17-26$ & $17-18$ \\
\hline 7:40 & & & & & $4-5$ & $20-21$ & $17-26$ & $17-18$ \\
\hline 7:50 & & & & & $4-5$ & $20-21$ & & $17-18$ \\
\hline 8:00 & & & & & $4-5$ & $5-20$ & & $17-18$ \\
\hline 8:10 & & & & & $5-6$ & $5-20$ & & $17-18$ \\
\hline 8:20 & & & & & $5-6$ & $5-20$ & & $17-26$ \\
\hline 8:30 & & & & & & $5-6$ & & $17-26$ \\
\hline 8:40 & & & & & & $5-6$ & & $17-26$ \\
\hline 8:50 & & & & & & $5-6$ & & \\
\hline
\end{tabular}




\section{CONCLUDING REMARKS}

In this paper, a concept for a taxi-movement-planning tool based on MILP models has been presented. Application of the tool results in a revision of the taxi plans for each aircraft to match the constraints while minimizing the "costs." The initial results are primarily related to a single planning update. Such an update involves the planning of all the current aircraft and those anticipated to be using the taxiway system within the planning horizon.

On the indication of the preliminary results, the concept appears to hold out great promise for further development. A real-life schedule and airport model will be implemented in the near future to demonstrate that the taxi-movementplanning tool is indeed able to optimally solve taxi planning scenarios, especially with respect to the complexity and size of the resulting MILP problems.

\section{REFERENCES}

[1] V. H. L. Cheng, V. Sharma, and D. C. Foyle, "A study of aircraft taxi performance for enhancing airport surface traffic control," IEEE Transactions on Intelligent Transportation Systems, vol. 2, no. 2, pp. 39-54, 2001.

[2] F. S. Hillier and G. J. Lieberman, Introduction to Operations Research, McGraw-Hill, Oakland, Calif, USA, 7th edition, 2001.

[3] ILOG, CPLEX release 9.1, 2005.

[4] J. W. Smeltink, M. J. Soomer, P. R. de Waal, and R. D. van der Mei, "An optimisation model for airport taxi scheduling," in Proceedings of the INFORMS Annual Meeting, Denver, Colo, USA, October 2004.

[5] D. E. Pitfield, A. S. Brooke, and E. A. Jerrard, "A MonteCarlo simulation of potentially conflicting ground movements at a new international airport," Journal of Air Transport Management, vol. 4, no. 1, pp. 3-9, 1998.

[6] J.-B. Gotteland, N. Durand, J.-M. Alliot, and E. Page, "Aircraft ground traffic optimization," in Proceedings of the 4th International Air Traffic Management R\&D Seminar (ATM '01), Santa Fe, NM, USA, December 2001.

[7] Á. G. Marín, "Airport management: taxi planning," Annals of Operations Research, vol. 143, no. 1, pp. 191-202, 2006.

[8] M. A. R. van Velthuizen, "Taxi planning optimization using mixed integer programming," Memorandum M-799, Faculty of Aerospace Engineering, Delft University of Technology, Delft, The Netherlands, June 1997. 

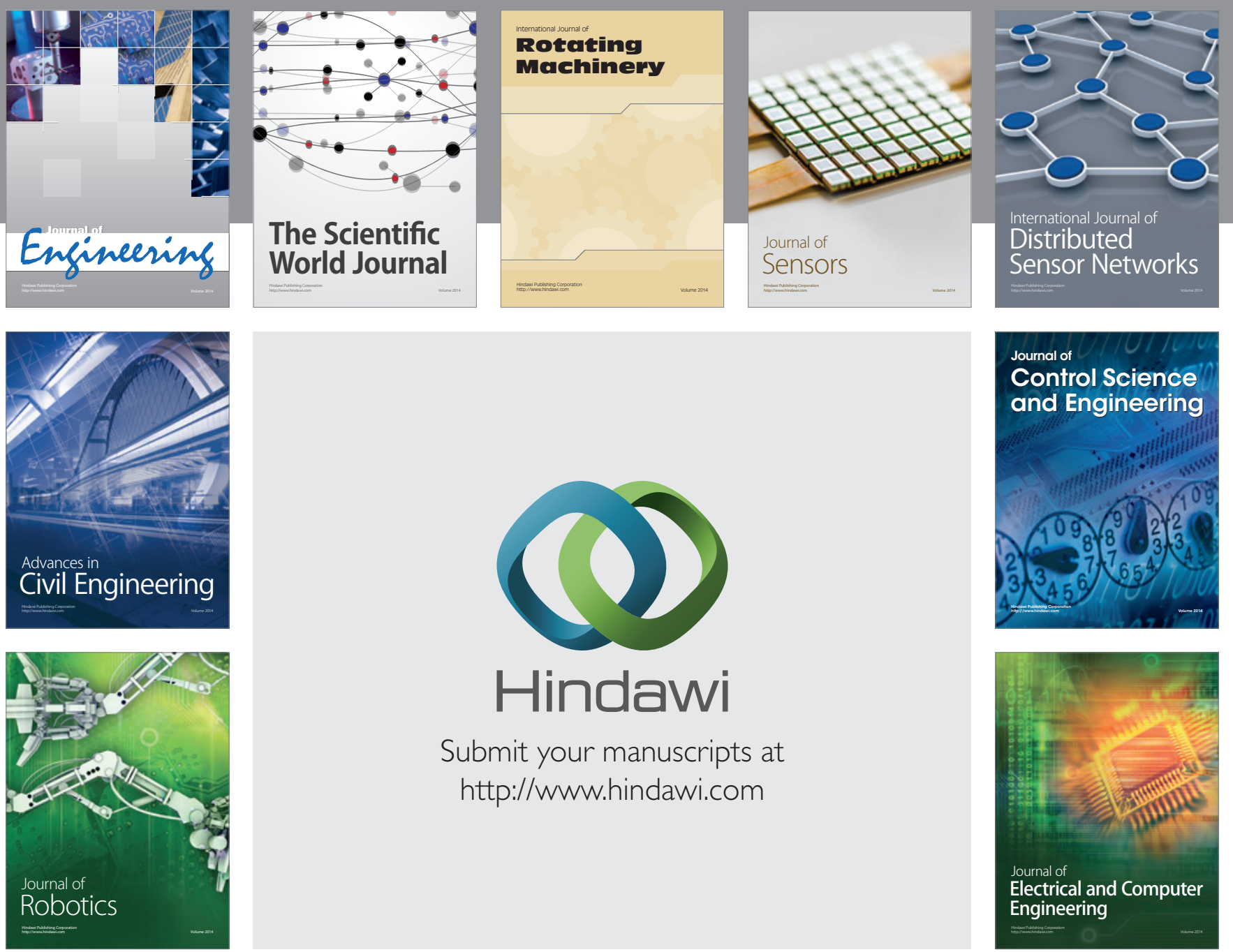

Submit your manuscripts at

http://www.hindawi.com
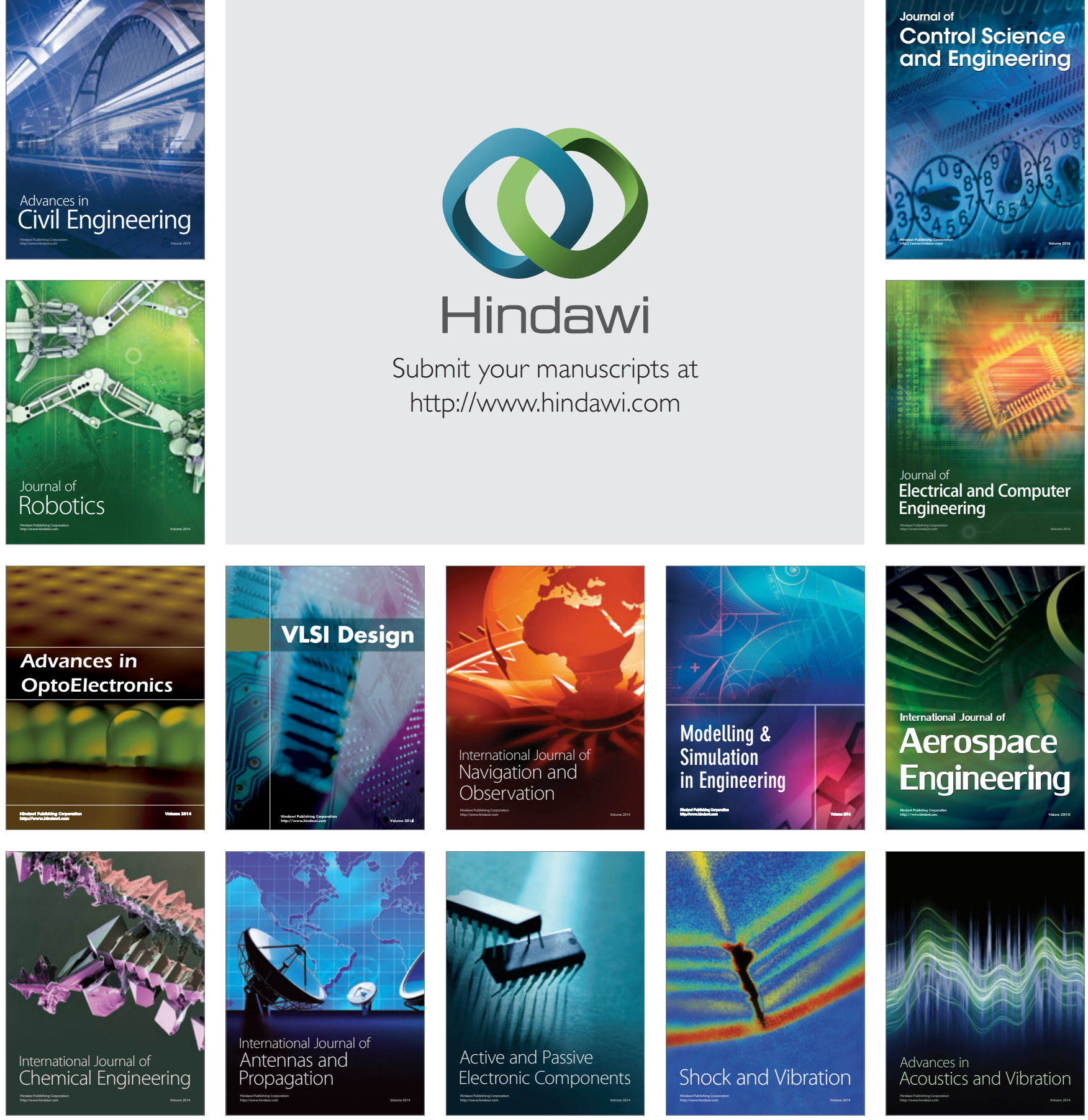